\title{
Research Paper \\ Comparison of Plantar Force, Pressure and Impulse During Walking in Men and Women With Flat Feet
}

\author{
Negin Soltani ${ }^{1}$ (), ${ }^{*}$ Ali Jalalvand ${ }^{1}$ (1), Mohammad Reza Jahani ${ }^{1}$ (1)
}

1. Department of Physical Education, Faculty of Humanities, Hamedan Branch, Islamic Azad University, Hamedan, Iran.

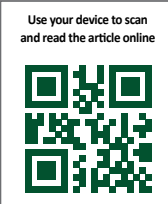

Cittation: Soltani N, Jalalvand A, Jahani MR. [Comparison of Plantar Force, Pressure and Impulse During Walking in Men and Women With Flat Feet (Persian)]. Journal of Sport Biomechanics. 2021; 7(1):94-107. https://doi.org/10.32598/biomechanics.7.2.2

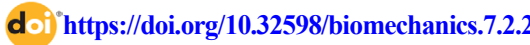

Keywords:

Plantar pressure distribution, Flat feet, Walking

\section{ABSTRACT}

Article Info:

Received: 23 Jun 2021

Accepted: 26 Jun 2021

Available Online: 01 Sep 2021

Objective This study aims to compare the variables of plantar force, pressure and impulse during walking in men and women with flat feet.

Methods The study population consists of non-athlete students with and without flat feet. Of these, 48 (male and female) were selected as study samples. The peak pressure, force and impulse on the foot were measured during walking by a foot scanner at a sampling frequency of $253 \mathrm{~Hz}$. Shapiro-Wilks test was used to examine the normality of data distribution, and data analysis was performed using MANOVA in SPSS software, considering the significance level at $P<0.05$.

Results Men with flat feet had more peak plantar pressure and force in the midfoot than healthy men, and more peak plantar pressure on the hallux. Women with flat feet had more peak plantar pressure and force on the hallux, toes T2-T3-T4-T5, M2 metatarsal head, and midfoot than healthy women. Men with flat feet had peak plantar pressure on the M4 metatarsal head than women with flat feet. Men with flat feet had different plantar impulses in the hallux, M2 metatarsal head, and lateral heal. Women with flat feet had more plantar impulses in the hallux, toes T2-T3-T4-T5, and midfoot than healthy women. There was a significant difference between men and women with flat feet in plantar impulses in metatarsal heads M3 and M4, midfoot, and lateral and medial heels

Conclusion Different effects of gender and sole structure on the distribution of plantar pressure should be considered in the production and design of shoes, medical insoles and special sports footwear.

\section{Extended Abstract}

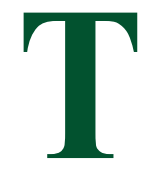

\section{Introduction}

he foot has a special structure; while providing a reliable level of support for standing, walking and moving, it bears a lot of pressure [1]. In order to stabilize and move the lower limb, the end of lower limb structures including the joints, ligaments, and muscles of the ankle and foot, are designed in such a way that can be able to bear the body weight with the least amount of energy while standing [2]. Foot deformity is one of the most common causes of pain, fatigue and dysfunction [4]. Flat foot is one of the most common foot deformities in the foot, which refers to loss of the medial longitudinal arch of the foot, and varies depending on the severity and amount of medial longitudinal arch loss.

One area that has recently attracted the attention of many researchers in the field of medicine and sports is the analysis of the distribution of plantar pressure. Measuring the plantar pressure distribution is one of the common methods

\section{* Corresponding Author:}

Ali Jalalvand, PhD.

Address: Department of Physical Education and Sport Sciences, Hamedan Branch, Islamic Azad University, Hamedan, Iran.

Tel: +98 (918) 9513172

E-mail: jalalvand_ali@yahoo.com 
Table 1. Comparing peak plantar pressure between men and women with and without flat feet

\begin{tabular}{|c|c|c|c|c|c|c|c|c|}
\hline \multirow{3}{*}{$\begin{array}{l}\text { Peak Plantar } \\
\text { Pressure }\end{array}$} & \multicolumn{4}{|c|}{ Mean \pm SD } & \multirow{3}{*}{ Sig.* } & \multirow{3}{*}{ Sig.** } & \multirow{3}{*}{ Sig.*** } & \multirow{3}{*}{ Sig.**** } \\
\hline & \multicolumn{2}{|c|}{ Men } & \multicolumn{2}{|c|}{ Women } & & & & \\
\hline & Flat Feet & Normal Feet & Flat Feet & Normal Feet & & & & \\
\hline Hallux & $4.82 \pm 4.02$ & $9.00 \pm 4.79$ & $5.80 \pm 0.89$ & $8.70 \pm 6.52$ & 0.020 & 0.033 & 0.884 & 0.855 .0 \\
\hline $\begin{array}{c}\text { Toe T2-T3- } \\
\text { T4-T5 }\end{array}$ & $2.70 \pm 1.66$ & $3.68 \pm 1.70$ & $2.38 \pm 1.63$ & $4.13 \pm 1.91$ & 0.120 & 0.032 & 0.842 & 0.444 \\
\hline M1 & $5.50 \pm 2.99$ & $6.72 \pm 3.02$ & $4.99 \pm 0.97$ & $6.24 \pm 3.72$ & 0.263 & 0.227 & 0.640 & 0.638 \\
\hline M2 & $8.79 \pm 4.75$ & $11.25 \pm 4.89$ & $7.29 \pm 1.98$ & $10.94 \pm 4.61$ & 0.121 & 0.048 & 0.590 & 0.833 \\
\hline M3 & $15.59 \pm 11.98$ & $19.42 \pm 8.02$ & $11.08 \pm 2.57$ & $14.61 \pm 4.75$ & 0.171 & 0.185 & 0.113 & 0.068 \\
\hline M4 & $15.68 \pm 14.17$ & $15.78 \pm 5.29$ & $12.65 \pm 3.05$ & $9.62 \pm 5.85$ & 0.974 & 0.297 & 0.327 & 0.033 \\
\hline M5 & $9.32 \pm 9.18$ & $7.97 \pm 3.73$ & $7.75 \pm 2.19$ & $6.13 \pm 5.72$ & 0.475 & 0.429 & 0.470 & 0.407 \\
\hline Midfoot & $4.24 \pm 1.77$ & $5.65 \pm 1.25$ & $3.98 \pm 0.69$ & $5.97 \pm 2.18$ & 0.038 & 0.001 & 0.673 & 0.483 \\
\hline Medial heel & $8.62 \pm 4.15$ & $9.11 \pm 3.91$ & $01.65 \pm 5.18$ & $8.24 \pm 3.20$ & 0.735 & 0.085 & 0.171 & 0.521 \\
\hline Lateral heal & $9.82 \pm 5.36$ & $8.96 \pm 2.37$ & $9.06 \pm 1.81$ & $8.69 \pm 4.34$ & 0.433 & 0.787 & 0.602 & 0.997 \\
\hline
\end{tabular}

*Comparison between men with and without flat feet; **Comparison between women with and without flat feet; ***Comparison between men and women with normal feet; $* * * *$ Comparison between men and women with flat feet.

that, while identifying the structural deformities of the foot, examines the performance of the foot in static and dynamic conditions, including walking [6]. Improper distribution of forces can cause abnormal movements and excessive stress and damage the tissues and muscles of the foot [8]. Center of pressure and peak pressure in different plantar areas are major factors in gait studies [9]. Foot plantar pressure analysis has opened up a new perspective on lower limb pathology. Many researchers have used the peak pressure on the foot sole in the stance phase of gait to find the causes of lower limb pain and injury [10]. In people with flat feet, due to the disturbance of the normal body alignment caused by the loss of arch, the amount and direction of the forces applied to the foot may change in different gait phases. Due to the fact that the foot is one of the most important parts of the body while walking, it has three functions: force absorption, ground contact, and transfer of propulsive forces [11]. Measuring the amount of force while walking has recently become a criterion for identifying or classifying people based on the pattern of their use of forces during walking [12]. Other kinetic parameter that is used to identify risk factors is the area under the reaction force curve (Impulse) [13]. Due to scant research on the comparison of plantar pressure variables such as force, pressure and impulse among men and women with flat feet, this study aims to compare the parameters of foot plantar pressure (ground reaction force, plantar pressure and impulse) in young people with flat feet while walking.

\section{Methods}

The study population consisted of non-athlete students with flat feet and normal feet. Of these, 48 were selected using a convenience sampling method. The peak plantar pressure, peak plantar force and impulse applied to the sole during walking were measured by a foot scanner at a sampling frequency of $253 \mathrm{~Hz}$. Shapiro-Wilks test was used to examine the normality of data distribution, and data analysis was performed using MANOVA in SPSS software, considering the significance level at $\mathrm{P}<0.05$.

\section{Results}

Men with flat feet had more peak plantar pressure and force in the midfoot than healthy men, and more peak plantar pressure on the hallux. Women with flat feet had more peak plantar pressure and force on the hallux, toes T2T3-T4-T5, M2 metatarsal head, and midfoot than healthy women. Men with flat feet had peak plantar pressure on the M4 metatarsal head than women with flat feet (Table 1). Men with flat feet had different plantar impulses in the hallux, M2 metatarsal head, and lateral heal. Women with flat feet had more plantar impulses in the hallux, toes T2- 
T3-T4-T5, and midfoot than healthy women. There was a significant difference between men and women with flat feet in plantar impulses in metatarsal heads M3 and M4, midfoot, and lateral and medial heels

\section{Discussion and Conclusion}

Different effects of gender and sole structure on the distribution of plantar pressure should be considered in the production and design of shoes, medical insoles and special sports footwear.

\section{Ethical Considerations}

Compliance with ethical guidelines

This study was approved by the Ethics Committee of Hamadan University of Medical Scieneces (Code: IR.UMSHA.REC.1398.256). All ethical principles are considered in this article. The participants were informed about the purpose of the research and its implementation stages. They were also assured about the confidentiality of their information. They were free to leave the study whenever they wished, and if desired, the research results would be available to them.

Funding

The paper was extracted from the MA. dissertation of the first author at the Department of Physical Education, Faculty of Humanities, Hamedan Branch, Islamic Azad University, Hamedan.

Authors' contributions

Conceptualization: Ali Jalalvand; Research: Ali Jalalvand, Negin Soltani; Editing and finalizing: All authors.

Conflicts of interest

The authors declared no conflict of interest. 
مقاله يخووهشى

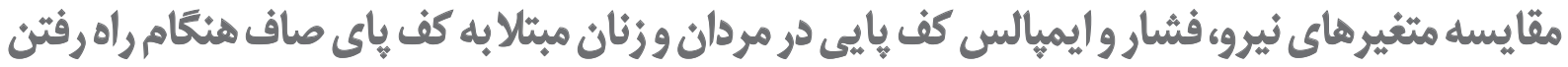

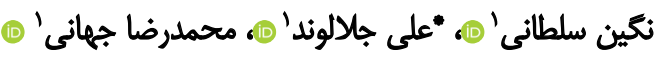

1. كروه تربيتبدنى، دانشكده علوم انسانى، واحد همدان، دانشعاه آزاد اسلامى، همدان، ايران.

\begin{abstract}
حكيد
هدف هدف از اين تحقيق مقايسه متغيرهاى نيرو، فشار وايميالس كف بايلي در مردان وزنان مبتلابه كف ياي صاف هئكام راه رفتن است.

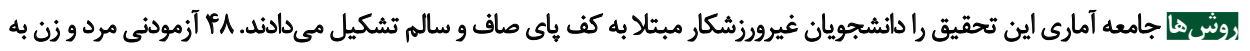

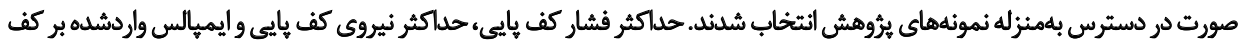

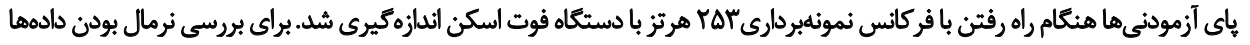

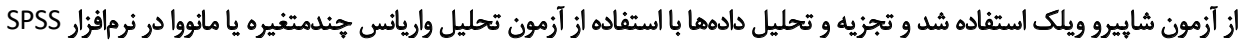

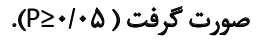

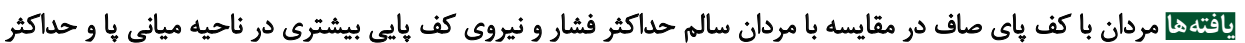

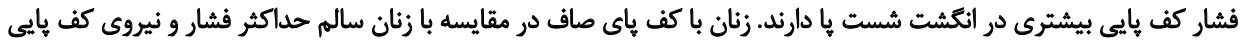

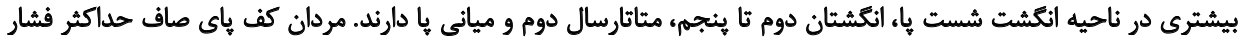

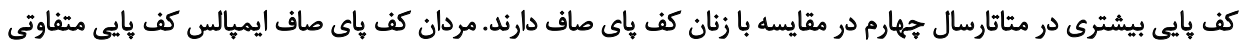

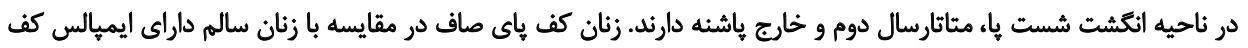

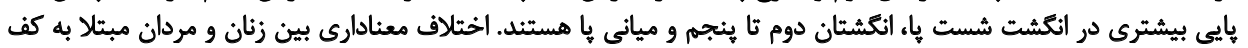

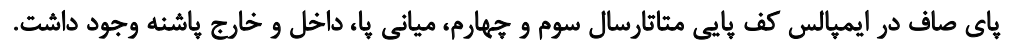

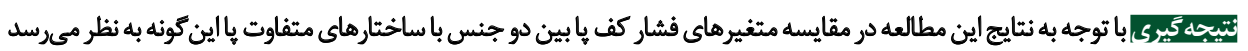

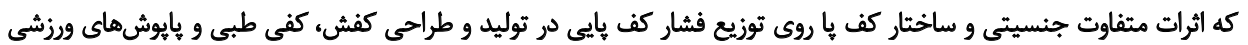

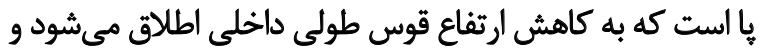

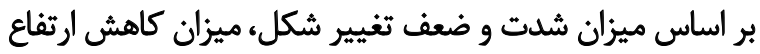

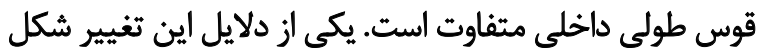

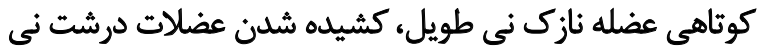

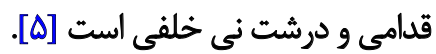
يا داراى ساختار ويرٔهاى است كه ضمن فراهم كردن سطح

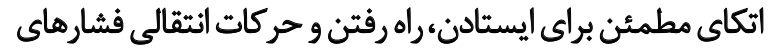

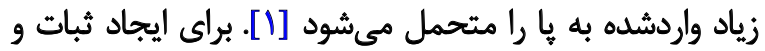

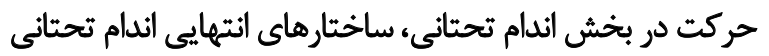

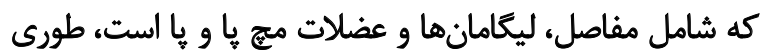

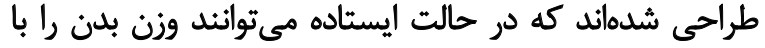

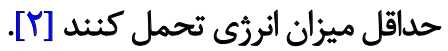

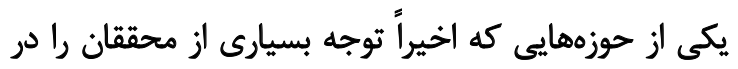

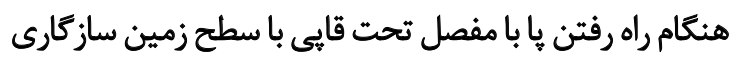




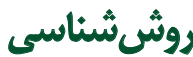

در اين مطالعه توصيفى مقايسهاي جامعه آمارى شامل

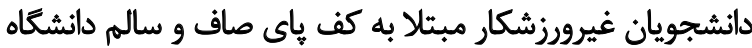

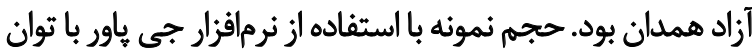

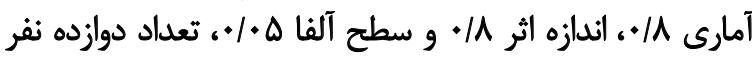

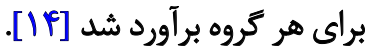

شركت كنندكان در دسترس كه شرايط ورود به مطالعه را دارا

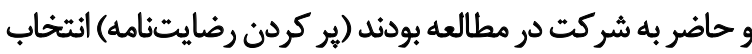

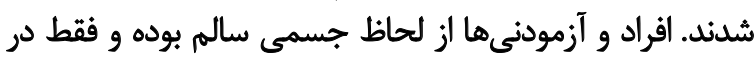

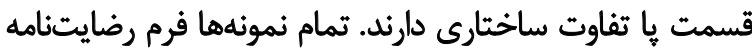

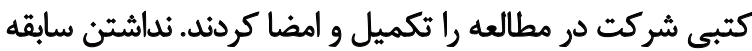

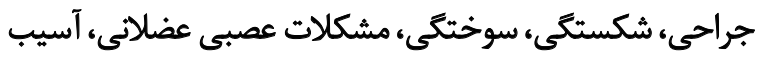

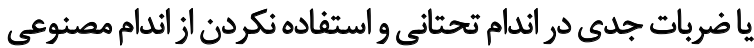

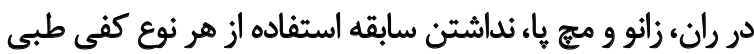

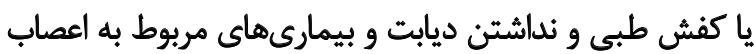
ي ييرامونى از شرايط عمومى آزمودنى ها بودي

آزمودنىها بر اساس ميزان شاخص نرمالايزشده ناوى به دو

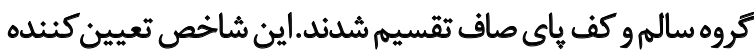

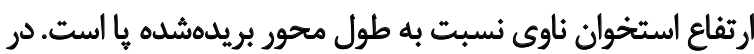

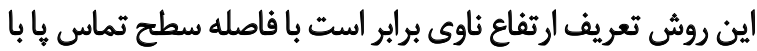

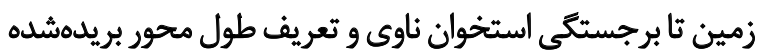

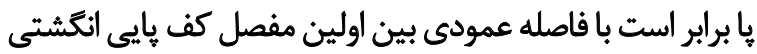

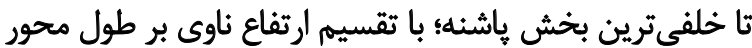

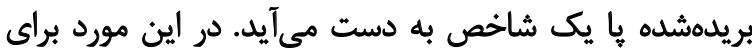

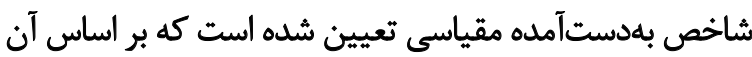

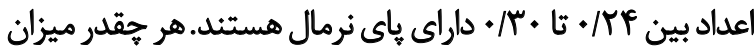

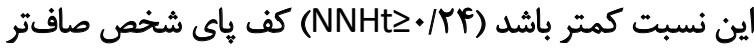

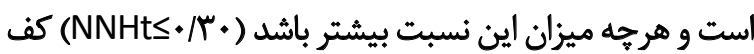

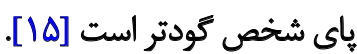

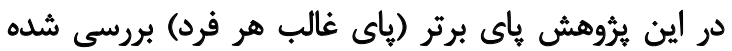

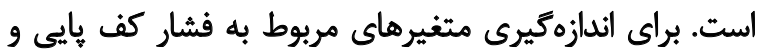

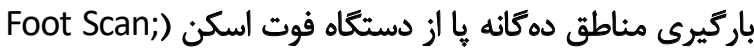
Foot Pressure

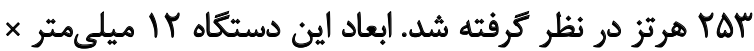

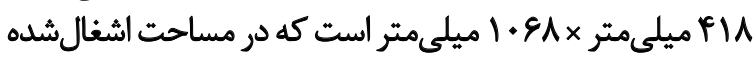

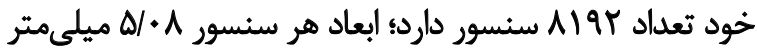

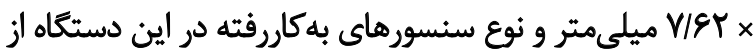

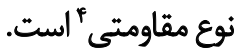

نواحى دهكانه با شامل انكشت شسته، انكُشتهاى دوم تأ

4. Resistive

5. Hallux

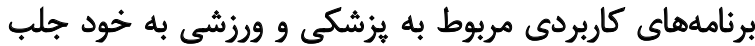

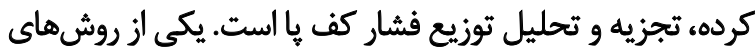

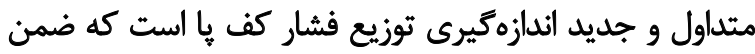

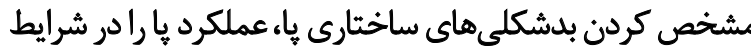

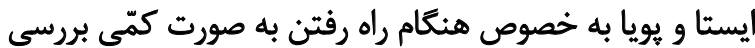

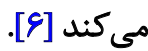

توزيع نامناسب نيروهاى كف ثاييى سبب ظهور حركات غيرطبيعى و اعمال استرس در ساختارهاى اندام تحتائى شئ شده

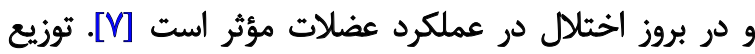

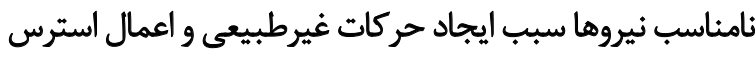

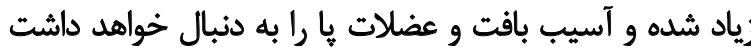

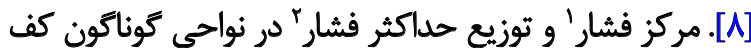

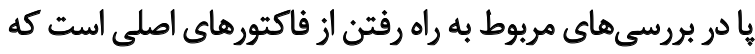

به آن توجه مي برشود [9].

تجزيهوتحليل فشار كف بايي،ديد إناهجديدى در آسيبشناسى

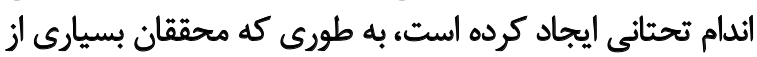

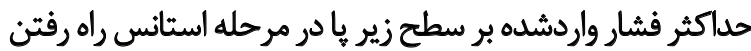

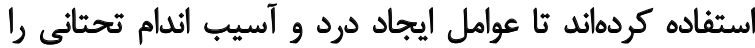

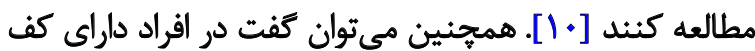

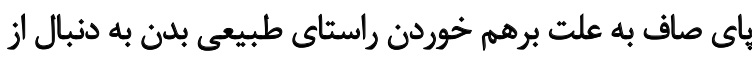

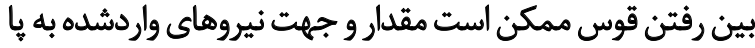

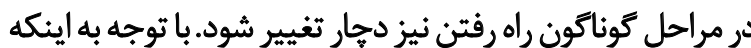

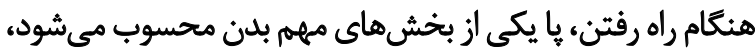

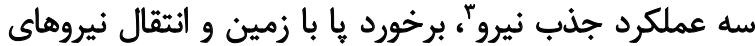

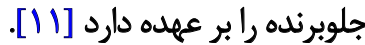

اندازهكيرى مقدار نيرو هنكام راه رفتن به تازگى معيارى براي

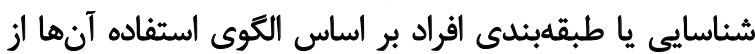

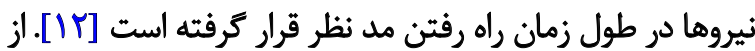

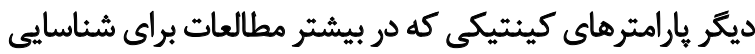

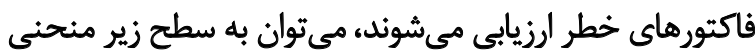

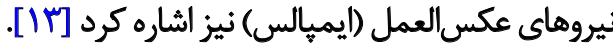

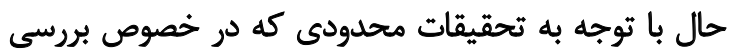

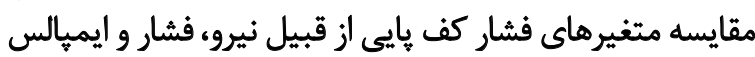

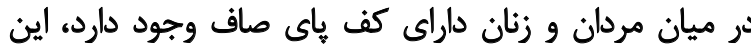

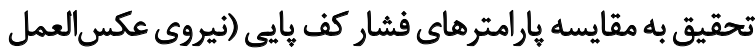

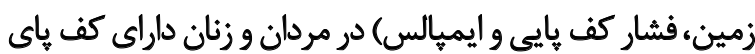
صاف هنكام راه رفتن مي يردازد.
1. Center of Pressure

2. Peak Pressure

3. Force 


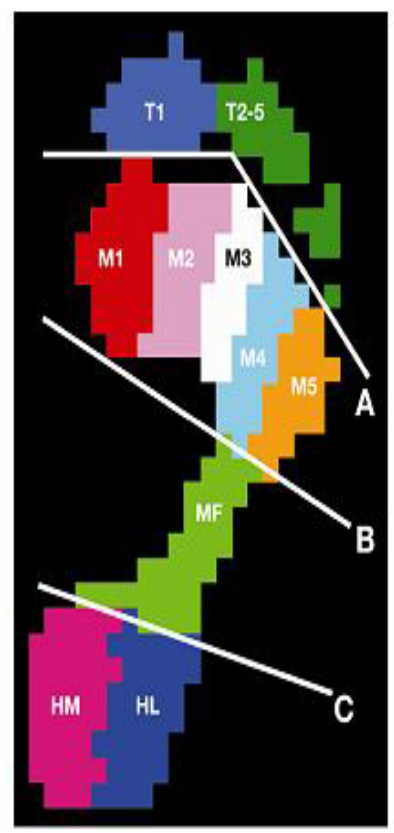

مجله بيومكانيك وزنثـ

آزمودني ها به صورت طبيعى جلو كيرى مي كرد. حتي از مترونوم

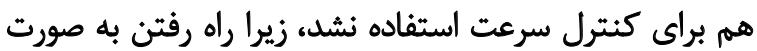

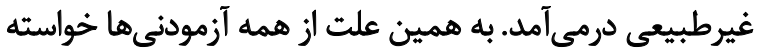

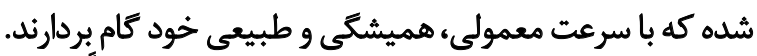

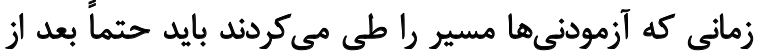

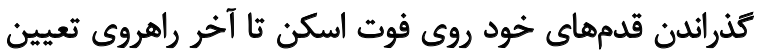

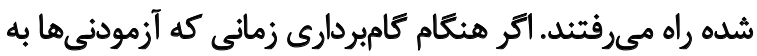

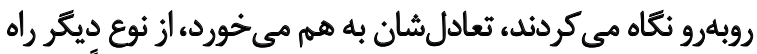

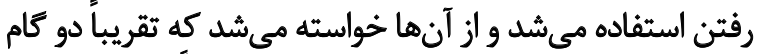

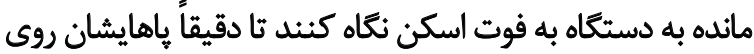
دستكاه فوت اسكن قرار بكيرد.

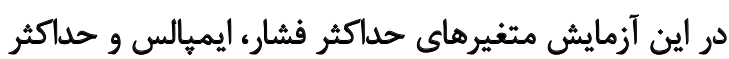

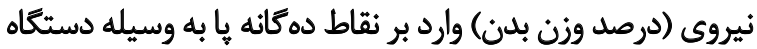

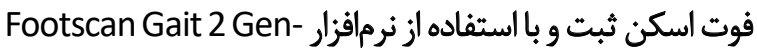
eration
تصوير ا. فشارهاى واردشده بر هاو تقسيمبئدى مثاطق دهكانه كف يا

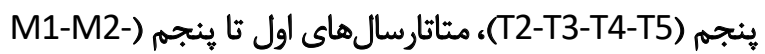

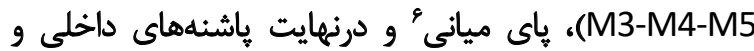

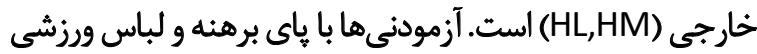

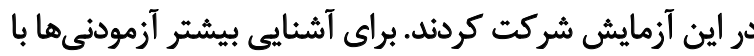

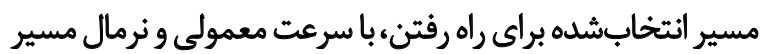

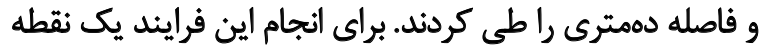

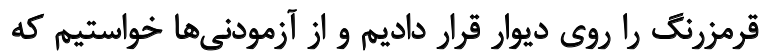

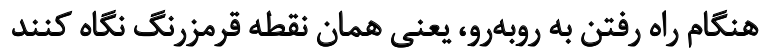

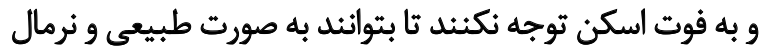

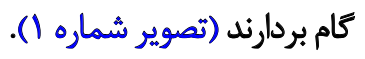

در اين يُروهش سرعت راه رفتن كنترل نشد، دليل اين امر

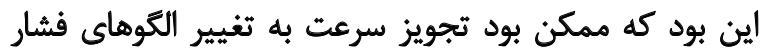

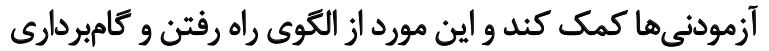

6. Midfoot

جدول ا. مشخصات جمعيتشناختى و شاخص افت ناوى در آزمودنىهاى مورد تحقيق

\begin{tabular}{|c|c|c|c|c|c|c|}
\hline \multicolumn{6}{|c|}{ كروهها } & \multirow{2}{*}{ مثغير } \\
\hline معنادارى & زنان كف باى صاف & زنان سالم & معادارى & مردان كف باى صاف & مردان سالم & \\
\hline . MIV & $r E / M T \pm T / \Delta A$ & $T r / T r \pm \Delta / 1 \mathcal{E}$ &.$/ 48 V$ & $T r / Y \Delta \pm 1 / T A$ & $r F / M \pm \Psi / \Delta \mid$ & سن (سال) \\
\hline ./MrT & $\mid \& \& / \& 9 \pm V / K r$ & $18.189 \pm \Delta / 18$ & - IETV & $|V q| \cdot \pm \pm q / n \mid$ & $1 W / W \pm 9 / 18$ & قد (سائتىمتر) \\
\hline 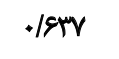 & SNEF士IV/FV & $\Leftrightarrow \Leftrightarrow / \mu^{\prime} \pm \Delta / v \Delta$ &.$/ 994$ & $V E / r \Delta \pm r / \lambda$ & $V E / T Y \pm Q / F i$ & جرم بلن (كيلوكرم) \\
\hline .1 .0 & $\cdot / r \cdot \pm \cdot / \cdot r$ & $\cdot|r V \pm \cdot| \cdot F \mid$ & $1+4$ & $. / 19 \pm \cdot / \bullet V$ & $. / K E \pm+/+4$ & شاخص افت ناوى \\
\hline
\end{tabular}


جدول r. مقايسه حداكثر فشار كف هايیى واردشده بر مناطق ده كانه بين مردان و زنان سالم و مبتلا به كف باى صاف

\begin{tabular}{|c|c|c|c|c|c|c|c|c|}
\hline \multirow{3}{*}{ 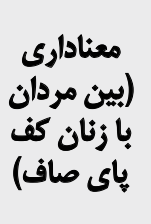 } & \multirow{3}{*}{ 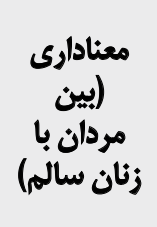 } & \multirow{3}{*}{ 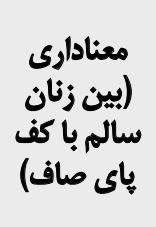 } & \multirow{3}{*}{ 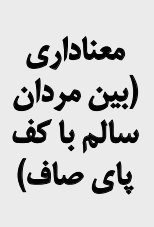 } & \multicolumn{4}{|c|}{ ميانكين+انحرافمعيار } & \multirow{3}{*}{ كداكثر فشار } \\
\hline & & & & \multicolumn{2}{|c|}{ زنان } & \multicolumn{2}{|c|}{ مردان } & \\
\hline & & & & سالم & كف باى & سالم & كف باى & \\
\hline.$/ 1 \Delta \Delta$ & - IMF & ז &.$+T_{\text {. }}$ & $N V+ \pm F / \Delta r$ & $\Delta / \cdot A \pm+/ Q \Lambda$ & $q / \cdots \pm f / v q$ & r/ATIY/.r & انكُشت شست \\
\hline.$/ P f f$ & . AAF & $.1 . \pi$ &.$/ N r$ & $f /(r \pm \mid / q)$ & $r / \Lambda \mu \pm \mid / r g$ & $r / \& \Lambda \pm 1 / V$. & $r / V+ \pm 1 / \mathscr{G}$ & انكُشتمبم دوم تا \\
\hline . & 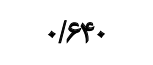 &.$/ \pi T$ & . & $g / \pi+\mu / N$ & $p / q q \pm+/ v q$ & $g / Y \Psi \pm / \cdot r$ & $\Delta / \Delta \cdot \pm r / 9 q$ & مثاتارسال اول \\
\hline זr/A & .109. & $.1 \cdot+4$ &.$/ M T$ & $1 . / 94 \pm+/ 81$ & V/QY \pm //R & $11 / r \Delta \pm F / A q$ & $N V q \pm F / V \Delta$ & متاتارسال دوم \\
\hline $.1+81$ &.$M H$ & . /Ma &.$/ 1 n$ & $|r / \varepsilon| \pm F / V \Delta$ & $11 / \cdot A \pm r / \Delta V$ & $I Q / F T \pm N \cdot r$ & $|\alpha / \Delta q \pm I| / Q \Lambda$ & مثاثارسال سوم \\
\hline r &.$/ M T V$ & . /rav &.$/ 9 h^{7}$ & V/gY $\pm \Delta / \wedge \Delta$ & $\mid r / s \Delta \pm r / * \Delta$ & $1 ه / V A \pm \Delta / q T$ & $|ه / \& A \pm| F / / V$ & متاثارسال \\
\hline$\cdot / r \cdot v$ & . IFr & . prqq & - IFVA & $g / N+\Delta / M$ & $V / V \Delta \pm r / / q$ & $v / v q \pm r / r v$ & Q/Tr士Q//A & مثاتارسال بنجم \\
\hline - MAN & $.18 x^{\circ}$ & $.1 \cdot+1$ & . 1 A & $\Delta / q \checkmark \pm r / M \Lambda$ & $r / q u+/ e q$ & $\Delta / \Delta \& \pm V / \Delta r$ & P/MPII/W & وسط با \\
\hline . $/ \Delta T \mid$ &.$/ N n$ & $.1 \cdot 1 \Delta$ & ./Mra & $N M T \pm T / Y$. & $1+/ 8 \Delta \pm \Delta / M$ & $9 / 11 \pm r / 19$ & NGY $\pm F / 1 \Delta$ & داخل باششئ \\
\hline.$/ 994$ & $.18 \cdot r$ & - /var & ./FTr & $N G q \pm F / m F$ & $\vee \cdot 9 \pm 1 / \wedge 1$ & $N g q \pm r / N$ & Q/AY $\pm \Delta / r q$ & خارج ياششئ \\
\hline
\end{tabular}

جدول با. مقايسه حداكثر نيروى كف هايي واردشده بر مناطق دهكائه بين مردان و زنان سالم و مبتّلا به كف ياي صاف

\begin{tabular}{|c|c|c|c|c|c|c|c|c|}
\hline \multirow{3}{*}{ 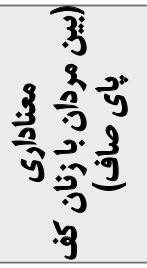 } & \multirow{3}{*}{ 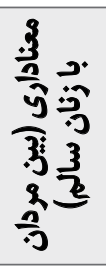 } & \multirow{3}{*}{ 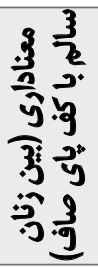 } & \multirow{3}{*}{ 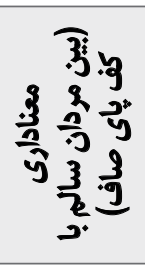 } & \multicolumn{4}{|c|}{ هيانكين|نحرافمعيار } & \multirow{3}{*}{ 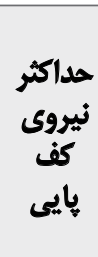 } \\
\hline & & & & \multicolumn{2}{|c|}{ زنان } & \multicolumn{2}{|c|}{ مردان } & \\
\hline & & & & سالم & كف باى صاف & سالم & كف باى صاف & \\
\hline$+M \cdot r$ &.$/ 4 E q$ & $+1+\cdot V$ & $\cdot M M$ & ITNAH $\pm 1+8 / 99$ & $\Delta \mathcal{N} / A \pm 1 \Delta / \wedge 9$ & $A V / F \Delta \pm \Gamma T / Y \Lambda$ & $V N+r \pm \varepsilon q / A T$ & شست بإ \\
\hline.$/ \Delta 10$ &.$/ 49$ & $.1 \cdot 11$ & 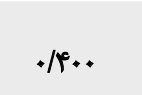 & $q V / . . \pm r q / \Delta$. & $P r / \circ 9 \pm 19 / 99$ & $8 . / 40 \pm 19 / 99$ & $\Delta 1 / T A \pm F T / 91$ & دور نان \\
\hline . /orq &.$/ r e 1$ &.$/ 149$ & $.11 \cdot 8$ & $91 / \Gamma \cdot \pm e q / V \Delta$ & EQ/EY $\pm|Y / A|$ & Vq/OYIPNIF & $A P / \Delta Y \pm S I / M^{P}$ & مثاثار ينجمل \\
\hline.$/ \Delta \Delta F$ &.$/ 1 \Delta F$ & $+1 \cdot \cdot V$ & (14) & Ire/Yo土eV/Fq & VNA. \pm r $/ / A r$ & IHF/TA土DWVE & $11 \cdot / 1 r \pm e q / 1 A$ & مثاتاربسال \\
\hline + $/ 4 M I$ & \% Ar & +1.90 & . $/ 4+q$ & $11 q / \mu+ \pm 8 V / V$ & $9 r / M \pm \pm r \cdot / M A$ & $W W q V \pm 1 \cdot q /+f$ & IET/Ar $\pm I F V / I V$ & مثاتارعسال \\
\hline .1 .99 &.$/ 1 \Delta \Delta$ & - leir & $\cdot N \cdot r$ & १९/9. $\pm 8 / / 1 \mathrm{~V}$ & $11 f / 9 V \pm F / .8$ & IFNV. $\pm \Delta r / 9 F$ & $18 \cdot 188 \pm 1 F \Delta / F 8$ & متاتارسال \\
\hline - IDAF &.$/ 958$ & - Ne8 & . Mes & $W / M=1 \cdot / 91$ & Ar/Aq土rf/rq & NAF $\pm F M$ & A)/AF $\pm \Delta V / 81$ & مثنائرالمبال \\
\hline$. / T \Delta \mid$ &.$/ D A A$ &. & $1+\cdots 1$ & $M|\Delta /| f \pm \mid f T / M I$ & IFNAF士FQ/.F & $r A \cdot / R f \pm 1 \mid \& / \& A$ & $\ln / \mathrm{r} \cdot \pm \mathrm{rq} / \mathrm{fr}$ & ويط يا \\
\hline.$/ \Delta 11$ & $+/ 499$ & $+/ T I M$ &.$/ 9 M 1$ & $I F / \Delta s \pm \Delta q / W$ & $|\& N \Delta Q \pm A V / F|$ & $\mid \Delta r / K E \pm \Delta F / F A$ & $10+199 \pm \lambda+199$ & هاخله \\
\hline - 1191 & $\cdot / M 11$ & + &.$/ M r$. & ITI/HIDNIF & $\mid r F / F V \pm r+/ A r$ & WWOF \pm HE/Fr & $10 \cdot / 7 \cdot \pm V q / T 8$ & خانهاج \\
\hline
\end{tabular}


از طرفي نتايج حاصل از مقايسه حداكثر فشار كف هايیى نقاط

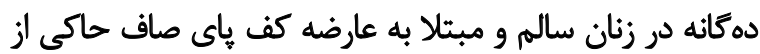

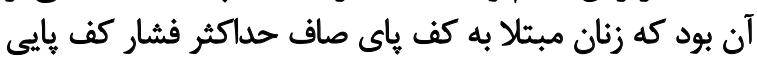

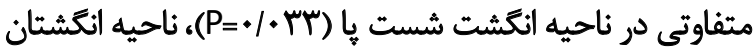

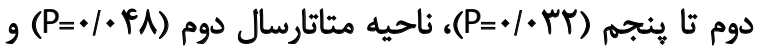

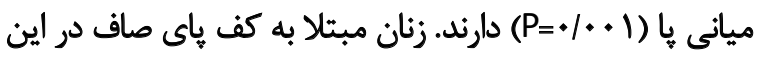

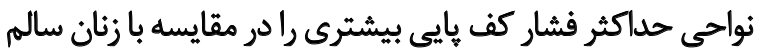

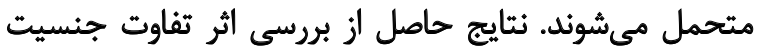

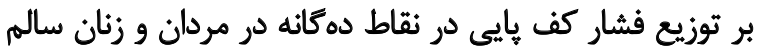

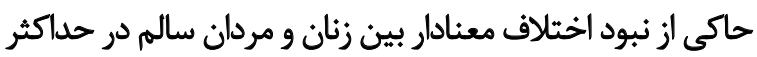

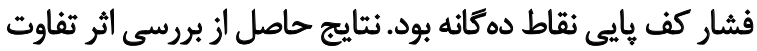

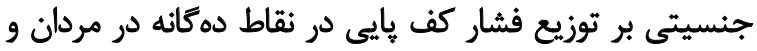

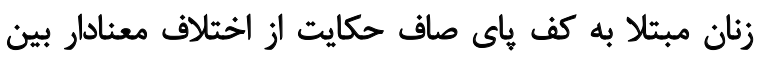

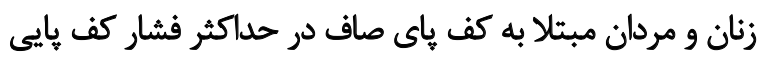

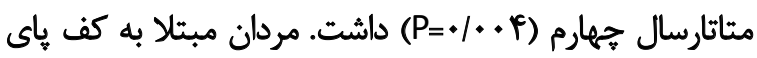

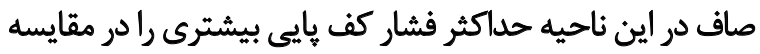

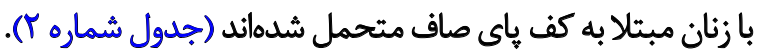
نتايج حاصل از مقايسه حداكثر نيروى كف باييى نقاط دهانهانه

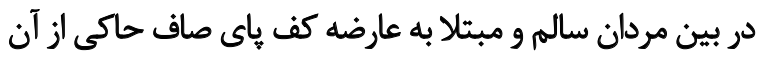

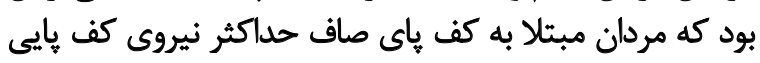

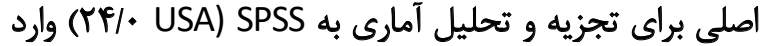

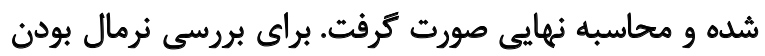

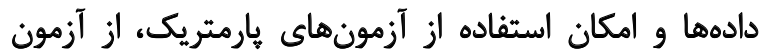

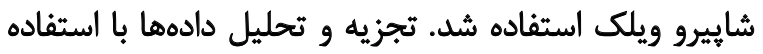

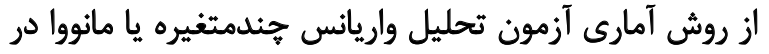

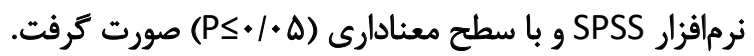

\section{نتايج}

مشخصات آزمودنى هاي شركت كنثله در اين يُروهش در جدول

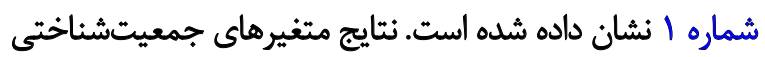

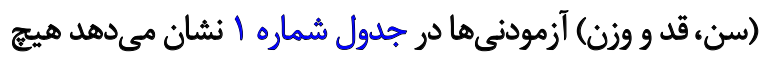

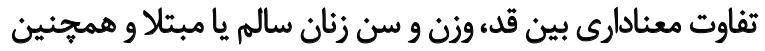

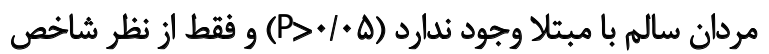

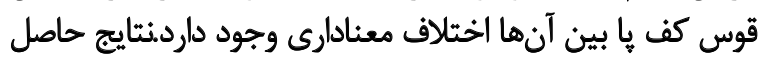

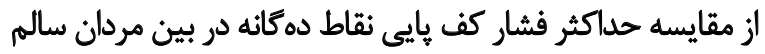

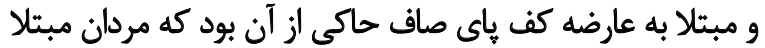

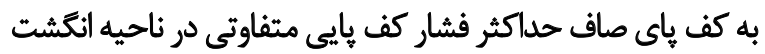

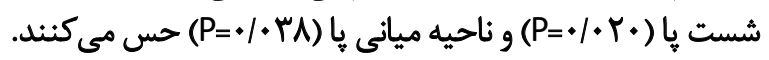

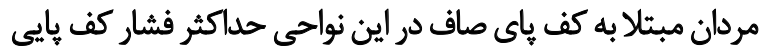

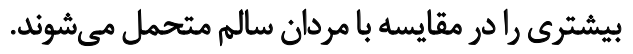

جدول f. مقايسه ايميالس واردشده بر مثاطق ده كائه هيا بين مردان و زنان سالم و مبثلا به كف باى صاف

\begin{tabular}{|c|c|c|c|c|c|c|c|c|}
\hline \multirow{3}{*}{ 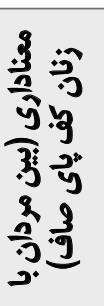 } & \multirow{3}{*}{ 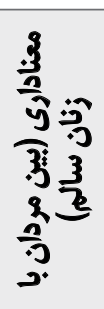 } & \multirow{3}{*}{ 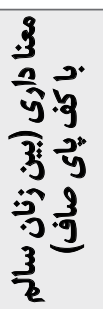 } & \multirow{3}{*}{ 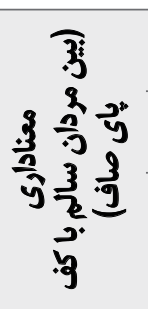 } & \multicolumn{4}{|c|}{ ميانكين ثانحرافمعيار } & \multirow{3}{*}{ ايميالس } \\
\hline & & & & \multicolumn{2}{|c|}{ زنان } & \multicolumn{2}{|c|}{ مردان } & \\
\hline & & & & سالم & كف إي صاف & سالم - & كف §اى صاف & \\
\hline$\cdot|A| r$ & - Neq & $.1 . . \mathrm{V}$ & .1 .14 & $r / m+1 / 4 q$ & $1 / / \vee \pm * / \ldots$ & r/PTII/IF & $1 / r \cdot \pm 1 / \%$ & أنكشت \\
\hline.$/ T A F^{6}$ & .|AP| & $.1 .0 \Delta$ & .1 .94 & $M A \pm \cdot / \Delta F \Delta$ & $\cdot / E q \cdot \pm \cdot /$ TAV & $V \cdot r \pm \cdot / r \cdot f$ & $\cdot M \pm \cdot / \Delta W$ & انأنشتان دوم \\
\hline.$/$ Mr & . $N \& A$ & -/ReA &.$/ M T r$ & $r / \mid F \pm 1 / M$ & $1 / M \pm \cdot / m 11$ & $r / f \& \pm \mid / P)$ & $r / \cdot . \pm 1 / T \cdot q$ & مثتاثارسال اول \\
\hline.$/ 4 \cdot 9$ &.$/ p 19$ & .1 .81 & $.1 \cdot F$ & $r / W \pm 1 / r r$ & $r / \Lambda \Delta \pm \cdot / \varphi+\Delta$ & $P / r Y \pm I / R$ & $r / T r \pm 1 / v e$ & مثاتارسال دوم \\
\hline$*+* 1$ &.$/ I M T$ &.$/ 814$ &.$/ N \Delta$ & $P / r \& \pm 1 / T r$ & $r / q+ \pm+(19)$ & $8 / V A \pm r / 19$ & $\Delta / r \varphi \pm r / A F$ & مثاتارسال \\
\hline .. TA & . /rAT &.$/$ TIF & . /AVQ & T/TA士I/Ne & $F / f e \pm 1 / F r$ & $\Delta / f \cdot \pm r / 1 q$ & $\Delta / \Delta \Delta \pm \Gamma / \Delta$ & هيأتارسال \\
\hline$\cdot / m$ &.$/ 909$ & . & . IAYE & $r / \cdot r \pm 1 / \& 8$ & $r / r \cdot \pm r / 19$ & MRVE./9RT & $r / / \& \pm r / . q$ & هثناثنارسال \\
\hline .1 .08 & - AAP & $.1 .0 r$ & $. / \Delta+$. & $r / r q \pm 1 / \cdot 1$ & I/prI士./pres & $1 / \Delta F \pm \cdot / \Delta \Delta A$ & I/RA土./\&AV & 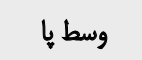 \\
\hline $.1 . \Delta$ & $.1 \cdot v^{8}$ &.$/ 199$ &.$/ M T A$ & $r(\Delta A \pm) / \cdot 1$ & $M / \cdot Y \pm V / \bullet \Delta$ & $1 / 9 T \pm \cdot 18 \Delta V$ & $r / r e \pm 1 / 10$ & داخل ياشئه \\
\hline $.1 \cdot .1$ & . Mme & $\cdot / \Delta \Delta \Delta$ & $.1 \cdot . v$ & r/PADI/MI & $r / \& A \pm \cdot / \Delta Y^{\prime}$ & V/DFI./MTA & $r / \Delta \Delta \pm / / \%$ & خارج هاشئه \\
\hline
\end{tabular}

مجله بيومكانيك وزنش 


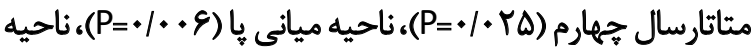

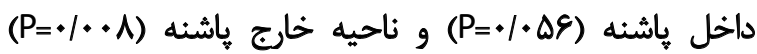

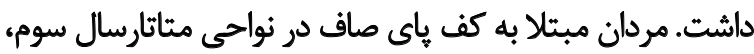

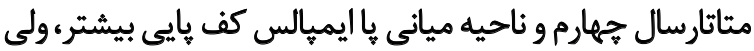

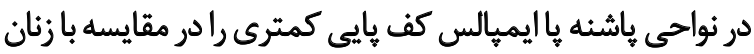
مبتلا به كف باى صاف متحمل شدهاند (جدول شماره f).

ب

هدف از اين يرؤهش مقايسه متغيرهاى نيرو، فشار و ايميالس

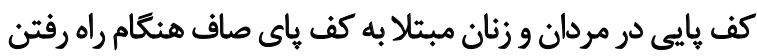

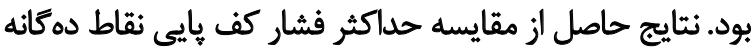

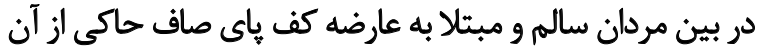

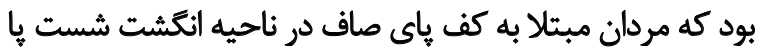

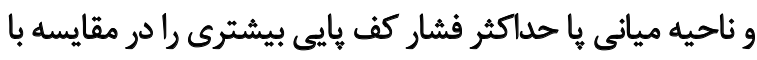

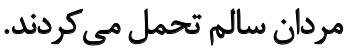

همجينين نتايج حاصل از مقايسه حداكثر فشار كف بائي نقاط

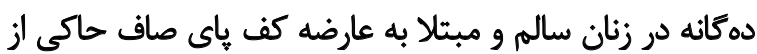

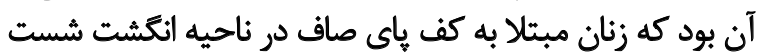

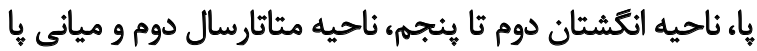

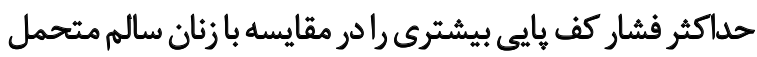

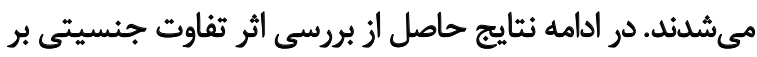

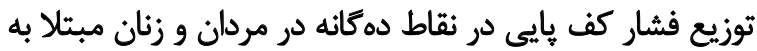

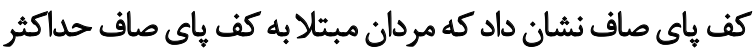

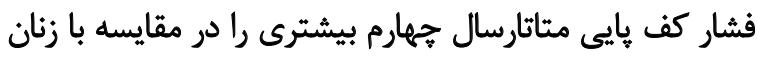
مبتلا به كف ياي صاف متحمل شدارساند.

ساير نتايج حاصل از مقايسه حداكثر نيروى كف باييى نقاط

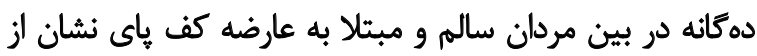

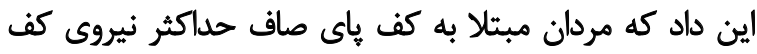

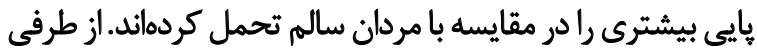

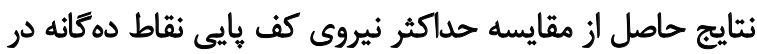

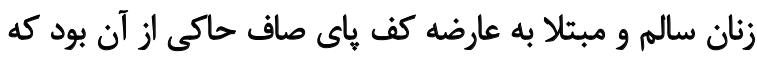

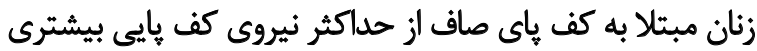

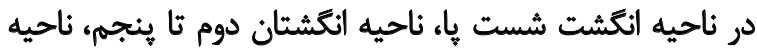

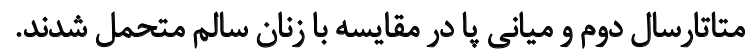

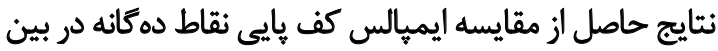

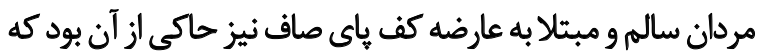

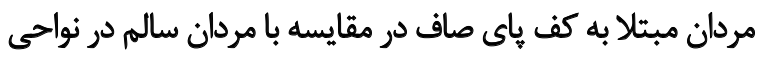

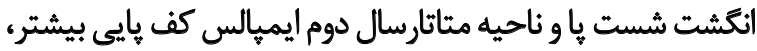
ولى در ناحيه خارج ياشنه ايميالس كف بائى كمترى دارند.

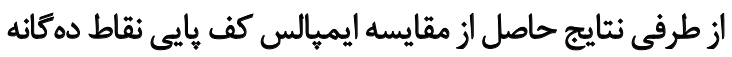

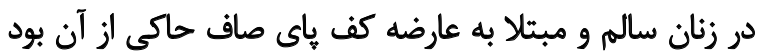

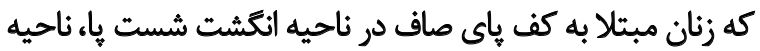

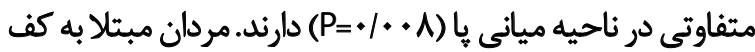

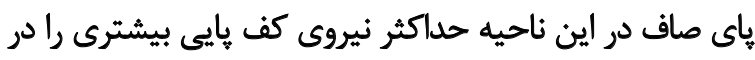

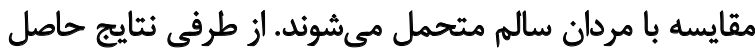

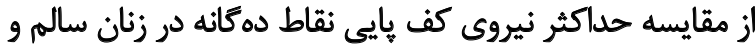

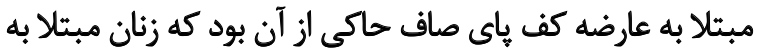

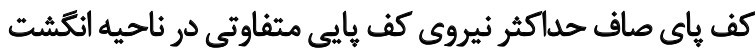

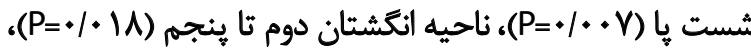

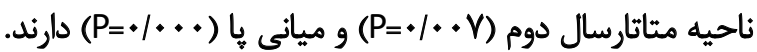

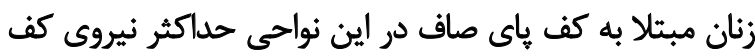

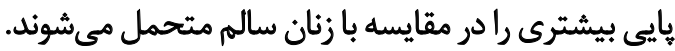

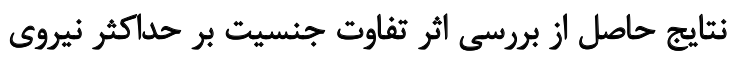

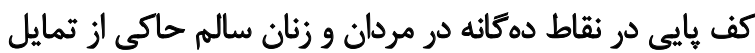

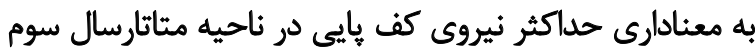

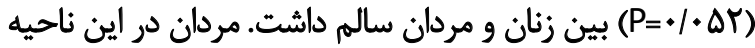

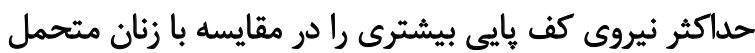
شداند (جدول شماره آ).

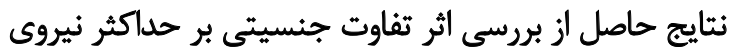

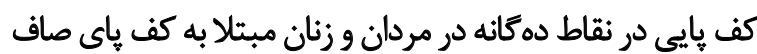

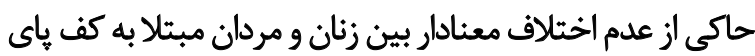
صاف در حداكثر نيروى كف إيايى نقاط دهأنانه بود.

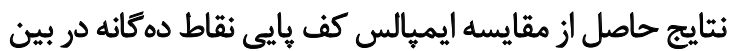

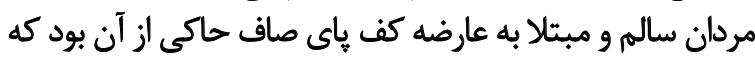

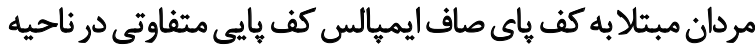

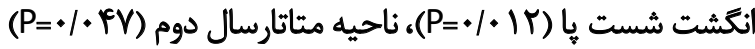

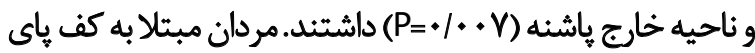

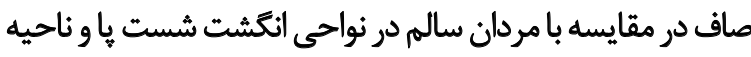

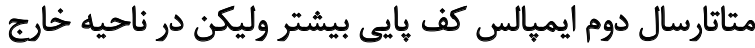
ياشنه ايميالس كف بايیى كمترى داشتند.

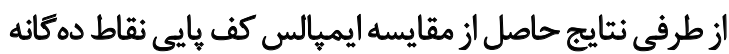

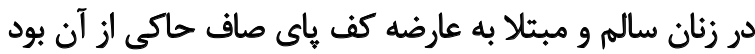

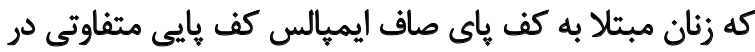

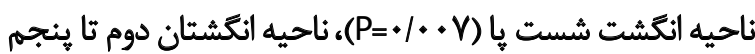

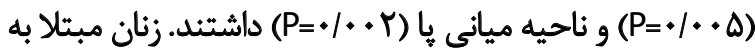

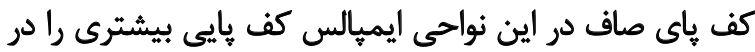

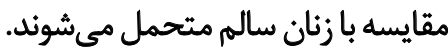

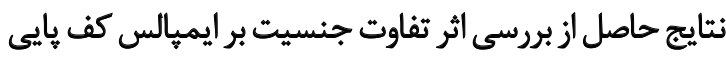

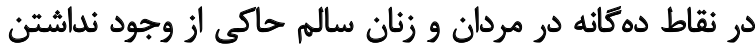

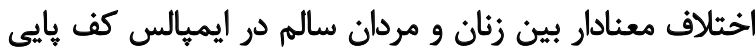

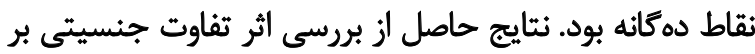

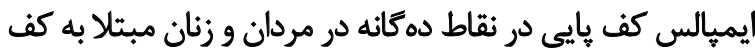

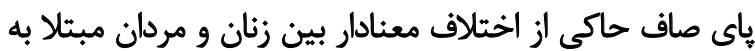

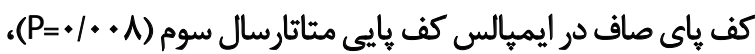


هم خوردن ريتم طبيعى و نرمال حركات هنكام راه رفتن مى شودود

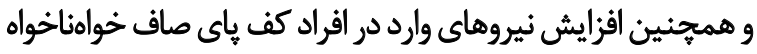

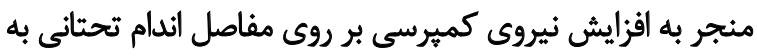
خصوص در قسمت مج و زانو مى شودي.

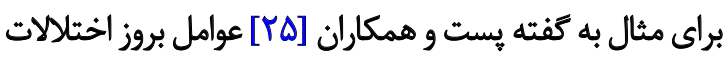

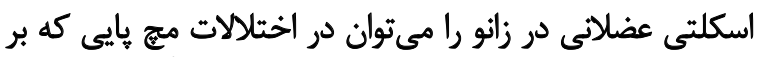

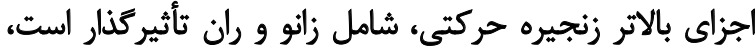

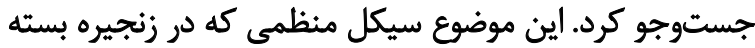

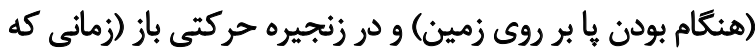

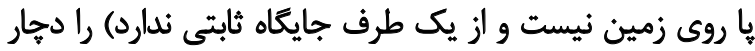

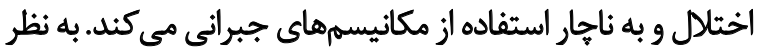

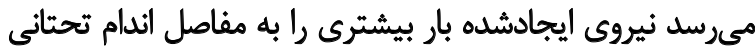

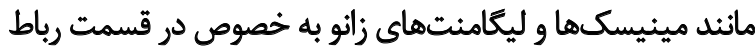

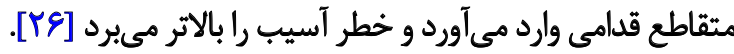

در ادامه نتايج اين يثروهش مشخص شد در زنان داراى صافى

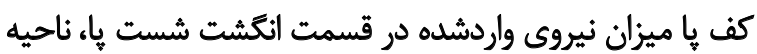

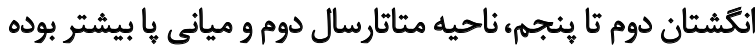

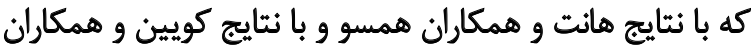

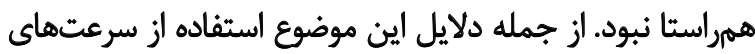

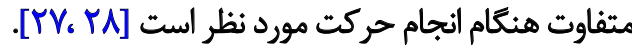

در مورد دلايل احتمالى مطرح براى اين افزايش نيرو در اين

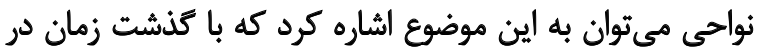

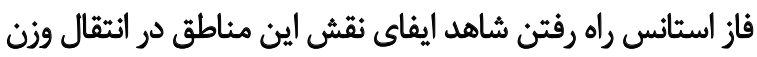

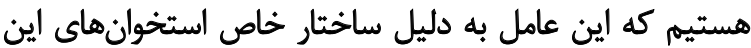

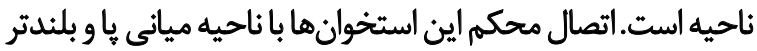

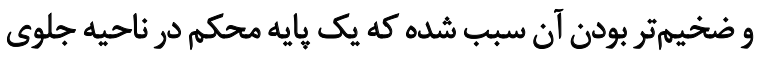

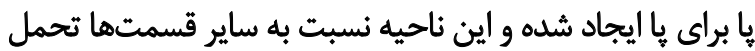

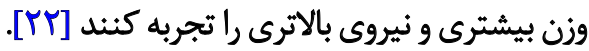

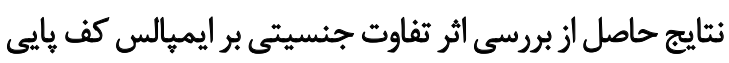

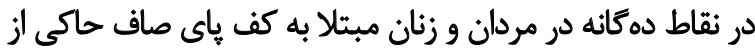

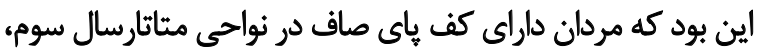

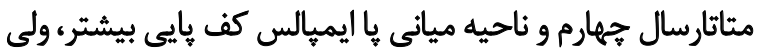

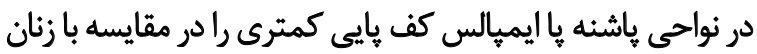

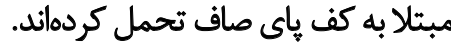

نتايج اين يُؤوهش با مطالعات كورنوال و همكاران همسو

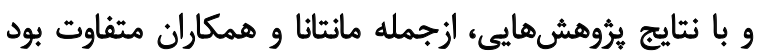

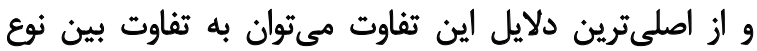

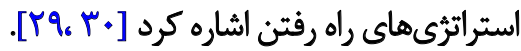

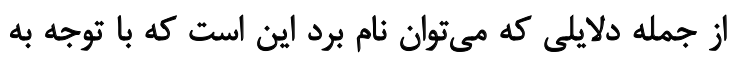

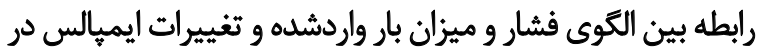

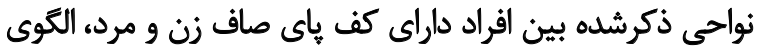

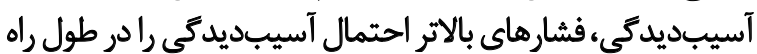

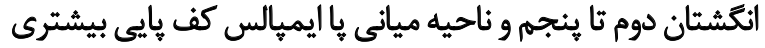

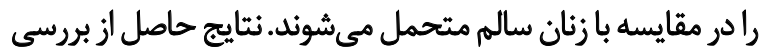

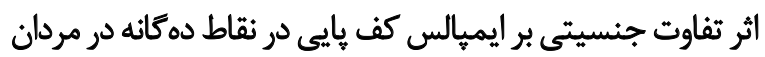

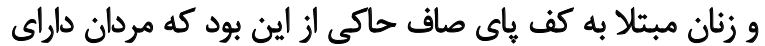

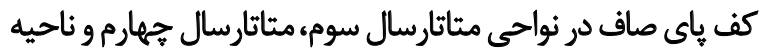

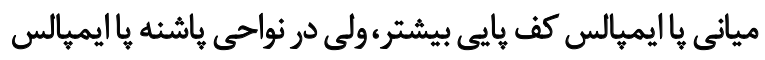

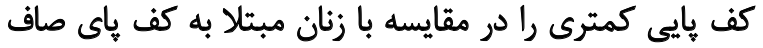
تحمل كردهاند. نتايج اين مطالعه با نتايج مطالعه جانلى و همكاران [19] همسو

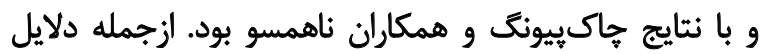

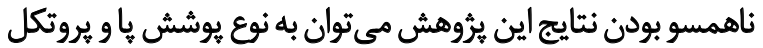

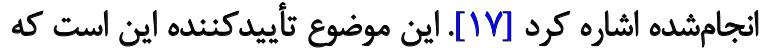

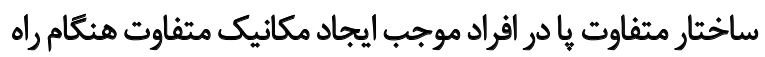

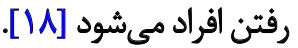

از جمله دلايل توجيهى و احتمالى براى اين موضوع اين است كه

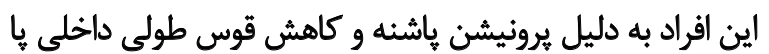

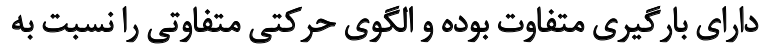

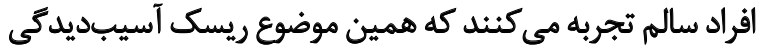

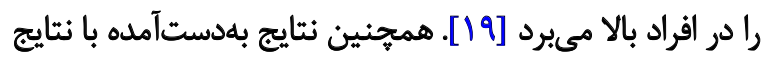

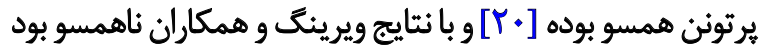

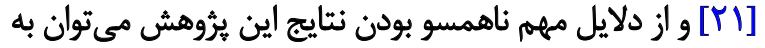

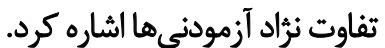

با توجه به بروهشهاى انجامشده تففاوت در نزاد در ساختار

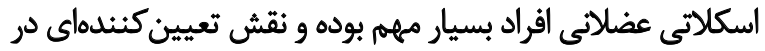

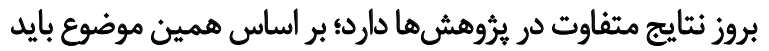

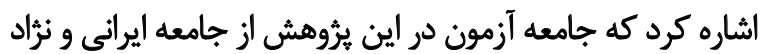

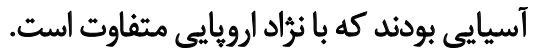

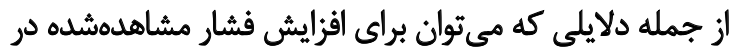

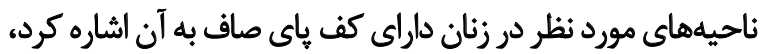

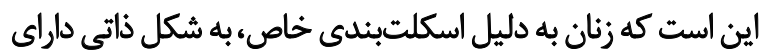

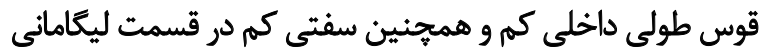

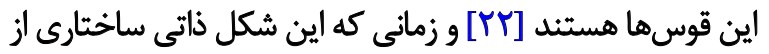

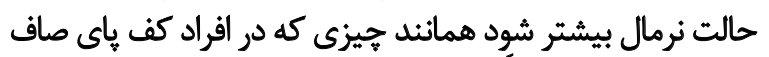

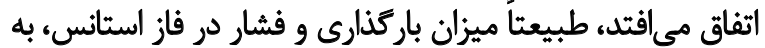

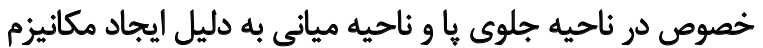

$$
\text { جبرانى بيشتر قابل مشاهده است. }
$$

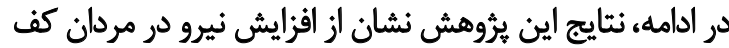

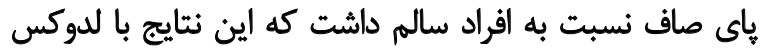

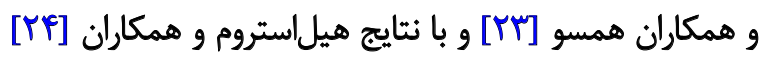

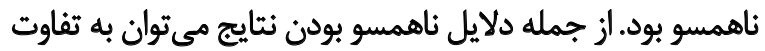

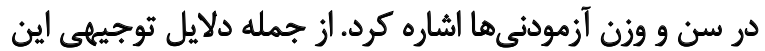

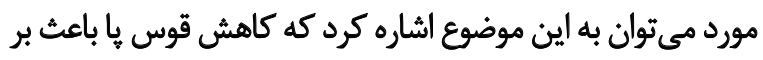




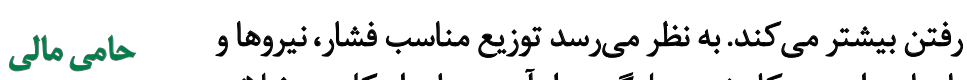

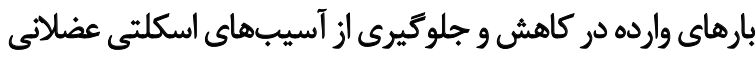
اين مقاله بخشى از إياننامه كارشناسى ارشد نويسنده اول در

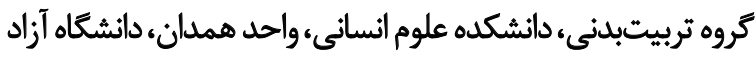

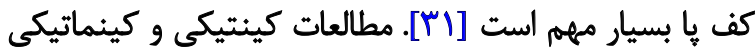

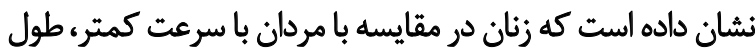

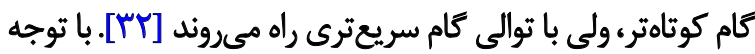

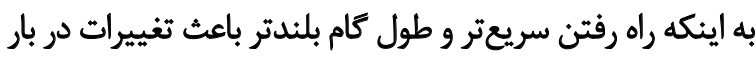
مشاركت نويسندكًان

مفهومسازى: على جلالوند؛ تحقيق و بررسى: على جلالوند،

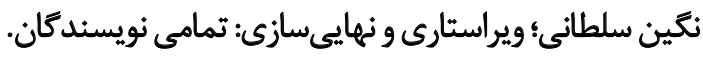

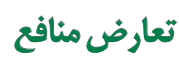

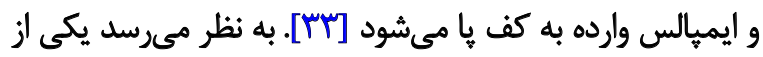

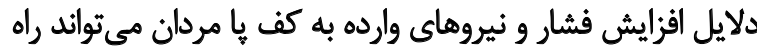
رفتن سريعتر و طول كام بلندتر در مقايسه با زنان باشد. با در نظر كرفتن اين امر كه بررسى حاضر در افراد بزركسال

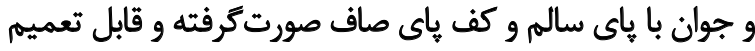
بنابر اظهار نويسندكان اين مقاله تعارض منافع ندارد.

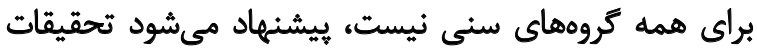

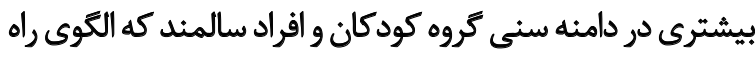
رفتن متفاوتى دارند و ممكن است دئن

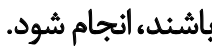

اين يثوهش نيز مائند ساير بثروهشهاى انجامشده

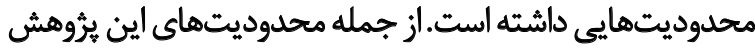

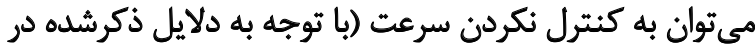

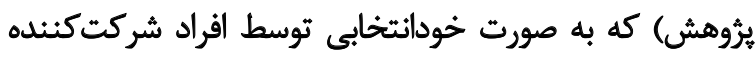

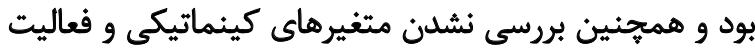

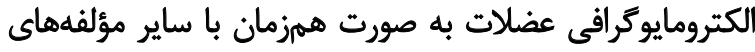
مورد بررسى در اين يُروهش اشاره كرد. تتيجلكيرى نهايي

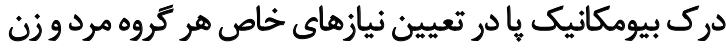

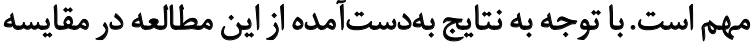

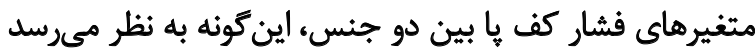

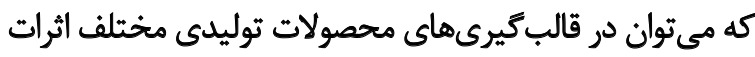

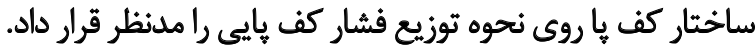

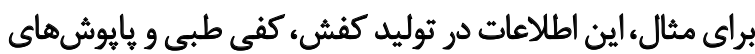

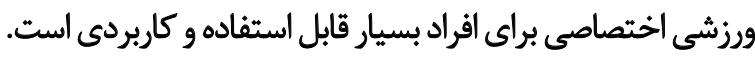

ملاحظات اخلاقى

$$
\text { يبيروى از اصول اخلاق يثوهش }
$$

اين يُروهش در كميته اخلاق يُروهش دانشكاه علوم يزشكى

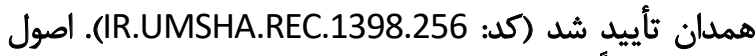
اخلاقي تماماً در اين مقاله رعايت شده است. شركت كنداند إندان

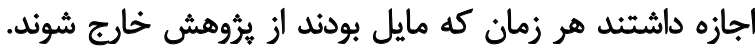

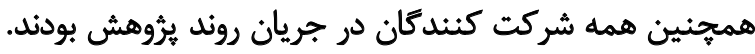
|طلاعات آن ها محرمائه نتكه داشته شدر. 


\section{Refrences}

[1] Levangie PK, Norkin CC. Joint structure and function: A comprehensive analysis. $5^{\text {th }}$ ed. Philadelphia: F.A. Davis Company; 2011. https://books. google.com/books?id=JXb2AAAAQBAJ\&printsec=frontcover\&dq

[2] Kisner C, Colby LA, Borstad J. Therapeutic exercise: Foundations and techniques. $7^{\text {th }}$ ed. Philadelphia: F.A. Davis Company; 2017. https:// books.google.com/books?id=yZc6DwAAQBAJ\&printsec=frontcover\&dq

[3] Bonato P, Ebenbichler GR, Roy SH, Lehr S, Posch M, Kollmitzer J, et al. Muscle fatigue and fatigue-related biomechanical changes during a cyclic lifting task. Spine. 2003; 28(16):1810-20. [DOI:10.1097/01. BRS.0000087500.70575.45] [PMID]

[4] Pauk J, Daunoraviciene K, Ihnatouski M, Griskevicius J, Raso JV. Analysis of the plantar pressure distribution in children with foot deformities. Acta Bioeng Biomech. 2010; 12(1):29-34. [PMID]

[5] Van Boerum DH, Sangeorzan BJ. Biomechanics and pathophysiology of flat foot. Foot Ankle Clin. 2003; 8(3):419-30. [DOI:10.1016/S10837515(03)00084-6] [PMID]

[6] Turner DE, Helliwell PS, Burton AK, Woodburn J. The relationship between passive range of motion and range of motion during gait and plantar pressure measurements. Diabet Med. 2007; 24(11):1240-6. [DOI:10.1111/j.1464-5491.2007.02233.x] [PMID]

[7] Kwon OY, Mueller MJ. Walking patterns used to reduce forefoot plantar pressures in people with diabetic neuropathies. Phys Ther. 2001; 81(2):828-35. [DOI:10.1093/ptj/81.2.828] [PMID]

[8] Safaei-Pour Z, Ebrahimi E, Saeedi H, Kamali M. [Invesigation of dynamic plantar pressure distribution in healthy adults during standing and walking (Persian)]. Arch Rehabil. 2009; 10(2):8-15. http://rehabilitationj.uswr.ac.ir/article-1-325-en.html

[9] Qu X, Yeo JC. Effects of load carriage and fatigue on gait characteristics. J Biomech. 2011; 44(7):1259-63. [DOI:10.1016/j.jbiomech.2011.02.016] [PMID]

[10] Nagel A, Fernholz F, Kibele C, Rosenbaum D. Long distance running increases plantar pressures beneath the metatarsal heads: A barefoot walking investigation of 200 marathon runners. Gait Posture. 2008; 27(1):152-5. [DOI:10.1016/j.gaitpost.2006.12.012] [PMID]

[11] Chui KC, Jorge M, Yen SC. Orthotics and prosthetics in rehabilitation. $4^{\text {th }}$ ed. Amsterdam: Elsevier Health Sciences; 2019. https:// books.google.com/books?id=ph4zwQEACAAJ\&dq

[12] Jenkins J, Ellis $C$. Using ground reaction forces from gait analysis: Body mass as a weak biometric. In: LaMarca A, Langheinrich $M$, Truong KN, editors. Pervasive Computing. Pervasive 2007. Lecture Notes in Computer Science. Vol. 4480. Berlin/Heidelberg: Springer. pp. 251-267. [DOI:10.1007/978-3-540-72037-9_15]

[13] Niu W, Feng T, Jiang C, Zhang M. Peak vertical ground reaction force during two-leg landing: A systematic review and mathematical modeling. Biomed Res Int. 2014; 2014:126860. [DOI:10.1155/2014/126860] [PMID] [PMCID]

[14] Faul F, Erdfelder E, Lang AG, Buchner A. G*Power 3: A flexible statistical power analysis program for the social, behavioral, and biomedical sciences. Behav Res Methods. 2007; 39(2):175-91. [DOI:10.3758/ BF03193146] [PMID]

[15] Murley GS, Menz HB, Landorf KB. A protocol for classifying normal-and flat-arched foot posture for research studies using clinical and radiographic measurements. J Foot Ankle Res. 2009; 2:22. [DOI:10.1186/1757-1146-2-22] [PMID] [PMCID]
[16] Jonely H, Brismée JM, Sizer Jr PS, James CR. Relationships between clinical measures of static foot posture and plantar pressure during static standing and walking. Clin Biomech. 2011; 26(8):873-9. [DOI:10.1016/j.clinbiomech.2011.04.008] [PMID]

[17] Chuckpaiwong B, Nunley JA, Mall NA, Queen RM. The effect of foot type on in-shoe plantar pressure during walking and running. Gait Posture. 2008; 28(3):405-11. [DOI:10.1016/j.gaitpost.2008.01.012] [PMID]

[18] Willems TM, De Ridder R, Roosen P. The effect of a long-distance run on plantar pressure distribution during running. Gait Posture. 2012; 35(3):405-9. [DOI:10.1016/j.gaitpost.2011.10.362] [PMID]

[19] Buldt AK, Murley GS, Butterworth P, Levinger P, Menz HB, Landorf $\mathrm{KB}$. The relationship between foot posture and lower limb kinematics during walking: A systematic review. Gait Posture. 2013, 38(3):363-72. [DOI:10.1016/j.gaitpost.2013.01.010] [PMID]

[20] Perttunen J. Foot loading in normal and pathological walking. Jyväskylä: University of Jyväskylä; 2002. https://books.google.com/ books?id=mWmzAAAACAAJ\&dq

[21] Wearing SC, Urry S, Smeathers JE, Battistutta D. A comparison of gait initiation and termination methods for obtaining plantar foot pressures. Gait Posture. 1999; 10(3):255-63. [DOI:10.1016/S0966 6362(99)00039-9] [PMID]

[22] Zifchock RA, Davis I, Hillstrom H, Song J. The effect of gender, age, and lateral dominance on arch height and arch stiffness. Foot Ankle Int. 2006; 27(5):367-72. [DOI:10.1177/107110070602700509]

[23] Ledoux WR, Hillstrom HJ. The distributed plantar vertical force of neutrally aligned and pes planus feet. Gait Posture. 2002; 15(1):1-9. [DOI:10.1016/S0966-6362(01)00165-5] [PMID]

[24] Hillstrom HJ, Song J, Kraszewski AP, Hafer JF, Mootanah R, Dufour $A B$, et al. Foot type biomechanics part 1: Structure and function of the asymptomatic foot. Gait Posture. 2013; 37(3):445-51. [DOI:10.1016/j.gaitpost.2012.09.007] [PMID] [PMCID]

[25] Post WR, Teitge R, Amis A. Patellofemoral malalignment: Looking beyond the viewbox. Clin Sports Med. 2002; 21(3):521-46 [DOI:10.1016/S0278-5919(02)00011-X] [PMID]

[26] Gokeler A, Hof AL, Arnold MP, Dijkstra PU, Postema K, Otten E. Abnormal landing strategies after $\mathrm{ACL}$ reconstruction. Scand J Med Sci Sports. 2010; 20(1):e12-9. [DOI:10.1111/j.1600-0838.2008.00873.x] [PMID]

[27] Hunt AE, Smith RM. Mechanics and control of the flat versus normal foot during the stance phase of walking. Clin Biomech. 2004; 19(4):391-7. [DOI:10.1016/j.clinbiomech.2003.12.010] [PMID]

[28] Queen RM, Mall NA, Nunley JA, Chuckpaiwong B. Differences in plantar loading between flat and normal feet during different athletic tasks. Gait Posture. 2009; 29(4):582-6. [DOI:10.1016/j.gaitpost.2008.12.010] [PMID]

[29] Cornwall MW, McPoil TG, Fishco WD, O'Donnell D, Hunt L, Lane C. The influence of first ray mobility on forefoot plantar pressure and hindfoot kinematics during walking. Foot Ankle Int. 2006; 27(7):53947. [DOI:10.1177/107110070602700710] [PMID]

[30] Mootanah R, Song J, Lenhoff MW, Hafer JF, Backus SI, Gagnon $D$, et al. Foot type biomechanics part 2: Are structure and anthropometrics related to function? Gait Posture. 2013; 37(3):452-6. [DOI:10.1016/j.gaitpost.2012.09.008] [PMID] [PMCID]

[31] Memar R, Noori S. [Comparison of plantar pressure distribution between the right and left foot and their correlation with height 
and weight at wrestlers (Persian)]. Res Sport Med Technol. 2016; 14(12):45-57. [DOI: 20.1001.1.22520708.1395.14.12.5.4]

[32] Cho SH, Park JM, Kwon OY. Gender differences in three dimensional gait analysis data from 98 healthy Korean adults. Clin Biomech. 2004; 19(2):145-52. [DOI:10.1016/j.clinbiomech.2003.10.003] [PMID]

[33] Sun D, Fekete G, Mei Q, Gu Y. The effect of walking speed on the foot inter-segment kinematics, ground reaction forces and lower limb joint moments. PeerJ. 2018; 6:e5517. [DOI:10.7717/peerj.5517] [PMID] [PMCID] 
This Page Intentionally Left Blank 